\title{
Prediction of antigenic epitopes on protein surfaces by consensus scoring Shide Liang ${ }^{1}$, Dandan Zheng ${ }^{2}$, Chi Zhang ${ }^{3}$ and Martin Zacharias*1,4
}

\author{
Address: ${ }^{1}$ School of Engineering and Science, Jacobs University Bremen, Campus Ring 1, D-28759 Bremen, Germany, ${ }^{2}$ Department of Radiation \\ Oncology, Massey Cancer Center, Virginia Commonwealth University, Richmond, VA, 23298, USA, ${ }^{3}$ The Center for Plant Science Innovation, \\ School of Biological Sciences, University of Nebraska, Lincoln, NE, 68588, USA and ${ }^{4}$ Physics Department, Technical University Munich, James \\ Franck Str. 1, D-85747 Garching, Germany \\ Email: Shide Liang - s.liang@jacobs-university.de; Dandan Zheng - Dandan.zheng@vcu.edu; Chi Zhang - chi.zhang@unl.edu; \\ Martin Zacharias* - m.zacharias@jacobs-university.de \\ * Corresponding author
}

Published: 22 September 2009

BMC Bioinformatics 2009, 10:302 doi:10.1186/147/-2105-10-302

This article is available from: http://www.biomedcentral.com/I47I-2/05//0/302

This is a

This is an Open Access article distributed under the terms of the Creative Commons Attribution License (http://creativecommons.org/licenses/by/2.0), which permits unrestricted use, distribution, and reproduction in any medium, provided the original work is properly cited.

Received: 7 April 2009

Accepted: 22 September 2009

\begin{abstract}
Background: Prediction of antigenic epitopes on protein surfaces is important for vaccine design. Most existing epitope prediction methods focus on protein sequences to predict continuous epitopes linear in sequence. Only a few structure-based epitope prediction algorithms are available and they have not yet shown satisfying performance.

Results: We present a new antigen Epitope Prediction method, which uses ConsEnsus Scoring (EPCES) from six different scoring functions - residue epitope propensity, conservation score, sidechain energy score, contact number, surface planarity score, and secondary structure composition. Applied to unbounded antigen structures from an independent test set, EPCES was able to predict antigenic eptitopes with $47.8 \%$ sensitivity, $69.5 \%$ specificity and an AUC value of 0.632 . The performance of the method is statistically similar to other published methods. The AUC value of EPCES is slightly higher compared to the best results of existing algorithms by about 0.034 .

Conclusion: Our work shows consensus scoring of multiple features has a better performance than any single term. The successful prediction is also due to the new score of residue epitope propensity based on atomic solvent accessibility.
\end{abstract}

\section{Background}

Realistic prediction of protein surface regions that are preferentially recognized by antibodies (antigenic epitopes) can help in the design of vaccine components and immuno-diagnostic reagents. Antigenic epitopes are classified as continuous or discontinues epitopes. If the residues involved in an epitope are contiguous in the polypeptide chain, this epitope is called a continuous epitope or a linear epitope. On the other hand, a discontinuous or non-linear epitope is composed of residues that are not necessarily continuous in the polypeptide sequence but have spatial proximity on the surface of a protein structure. A significant fraction of epitopes are discontinuous in the sense that antibody binding is not fully determined by a linear peptide segment but also influenced by adjacent surface regions [1].

However, the majority of available epitope prediction methods focus on continuous epitopes due to the convenience of the investigation in which the amino acid 
sequence of a protein is taken as the input. Such prediction methods are based upon the amino acid properties including hydrophilicity [2,3], solvent accessibility [4], secondary structure [5], flexibility $[6]$, and antigenicity [7]. In addition, based on the known linear epitope databases such as Bcipep [8] and FIMM [9], there also exist some methods using machine learning algorithms such as Hidden Markov Model (HMM) [10], Artificial Neural Network (ANN) [11], and Support Vector Machine (SVM) $[12,13]$ to locate linear epitopes. A study by Blythe and Flower has demonstrated that, using single-scale amino acid property profiles, a linear epitope prediction method was not able to predict epitope location reliably [14], whereas Greenbaum et al. showed that, using a combination of more than one amino aid property scale, machine learning algorithms could improve prediction accuracy[15].

Unlike linear epitope prediction, only a small number of studies have been performed so far on the prediction of discontinuous epitopes employing structural information of a target protein. Although such studies are of highly importance, the small number of available structures of antibody-antigen complexes limits this kind of studies. Several databases, such as IEDB [16], SACS [17], and CED [18], collected all existing structures of antibody-antigen complexes from the PDB bank. With the 3-dimentional structures of proteins as input, a few methods have been designed to predict putative antigenic epitopes by using conservation score, amino acid statistics, accessibility, and spatial information [19-24]. Ponomarenko and Bourne evaluated DiscoTope[20] and CEP[19] along with six other protein binding site prediction methods by benchmarking on 62 epitope structures and 82 antibody-antigen structures. They concluded that none of those prediction methods have a performance exceeding $40 \%$ precision and $46 \%$ recall[25]. Clearly, there is still a large gap between the strong need for antigenic epitope prediction and the low accuracy that existing prediction methods can achieve.

Use of multiple features could potentially improve performances on predicting antigenic epitopes, but this raises another question: are the properties effective for the limited number of antigens with available complex structures also work as well for all antigens? In this study, we tested 6 properties, which were used in protein/antibody binding site prediction previously, with the published databases plus the most recently released PDB entries. We found that the performances of the 6 terms were quite different for the two databases. Nevertheless, consensus prediction of the 6 terms resulted in reasonable accuracy for both databases.

\section{Methods \\ Protein datasets \\ Protein Dataset 1}

48 antigen-antibody complexes with resolution $<3.0 \AA$ were selected from the 59 representative antigen-antibody complexes compiled by Ponomarenko and Bourne[25]: $\underline{2 \mathrm{ADF}}, \underline{1 \mathrm{FE} 8}, \underline{1 \mathrm{BGX}}, \underline{1 \mathrm{EGI}}, \underline{1 \mathrm{EGI}}, \underline{\mathrm{FSK}}, \underline{\mathrm{H} 0 \mathrm{D}}, \underline{1 \mathrm{IQD}}$, $\underline{1 \mathrm{JRH}}, \underline{1 \mathrm{LK} 3}, \underline{1 \mathrm{MHP}}, \underline{1 \mathrm{NL}}, \underline{1 \mathrm{NSN}}, \underline{1 \mathrm{OAZ}}, \underline{1 \mathrm{ORS}}, \underline{\mathrm{PKQ}}$, 1RJL, 1SY6, 1TZI, 1WEI, 1YJD, 1YY9, 1ZTX, 2JEL, 1A14, $1 \mathrm{NCA}, 1 \mathrm{BVK}, 1 \mathrm{JHL}, 1 \mathrm{NDG}, 1 \mathrm{P} 2 \mathrm{C}, 1 \mathrm{JPS}, 1 \mathrm{AR} 1,1 \mathrm{EO} 8$, $1 \mathrm{QFU}, 1 \mathrm{EZV}, 1 \mathrm{OSP}, 1 \mathrm{FJ} 1,1 \mathrm{FNS}, 1 \mathrm{G} 9 \mathrm{M}, 1 \mathrm{R} 3 \mathrm{I}, 1 \mathrm{~N} 8 \mathrm{Z}$, $\underline{1 \mathrm{NFD}}, \underline{1 \mathrm{TQB}}, \underline{2 \mathrm{VDL}}, \underline{1 \mathrm{~V} 7 \mathrm{M}}, \underline{1 \mathrm{XIW}}, \underline{2 \mathrm{AEP}}$, and 1R0A. All entries were released before January 2006 except for $2 \mathrm{VDL}$, which was the new version of original entry $1 \mathrm{TXV}$. This dataset was used to derive residue epitope propensities.

\section{Protein Dataset 2}

22 antigen-antibody complexes and their unbound structures were selected from protein docking Benchmark 2.0 [26]. Benchmark 2.0 was published in 2005 and overlaps with Protein Dataset 1 . The complex structures in this dataset were used to locate the antibody binding sites. Interface residues on the surface of unbound antigens were used to optimize the parameters for the binding site prediction method and considered as the training set.

\section{Protein Dataset 3}

This dataset was curated by us and served as an independent test set, which has 17 antigen-antibody complexes released between February 2006 and October 2008. Within this window, there were 180 entries returned by querying the PDB with a resolution $<3.0 \AA$, using key words "antibody" and "complex". All complexes of antibodies with non-protein-ligands were manually removed from those 180 structures. Subsequently we performed a sequence alignment for antigens in the remaining complexes and Protein Dataset 1. A complex was kept if the maximum sequence identity between its antigen and any antigen in Protein Dataset 1 was less than 35\% in local alignment. For a complex with a maximum sequence identity in the range of $35 \sim 50 \%$, we accepted the complexes if the binding topology was not the same as the corresponding complex in Protein Dataset 1. The same criterion was also applied on any two complexes within Protein Dataset 3 itself. As a result, a total of 17 antigenantibody complexes were selected. The unbound structures of the antigens in those 17 complexes were also obtained from the PDB. The structure with the best resolution was selected if there was more than one protein structure available in PDB. For the case that an antigen's unbound structure was not available, its bound structure in a complex with another protein was used for evaluation. 


\section{Definition of surface residues, surface patches, and interface residues}

Following previous work [27], we consider an amino acid residue as a surface residue if the relative accessibility of its side chain is greater than $6 \%$ with probe radius $=1.2 \AA$. Also to confirm with previous work on protein binding site prediction [27] a surface patch is defined as a central surface residue and its 19 nearest surface neighbors in space. Solvent vector constraints [28] were applied in order to avoid patches sampled on different sides of a protein surface. An interface residue is the surface residue with solvent accessibility decreased more than $1 \AA^{2}$ upon association.

\section{Six terms for antibody binding site prediction}

Residue epitope propensity[29], conservation score[29], side chain energy score[29], contact number[20], surface planarity score[30], and secondary structure composition[5] were exploited for antibody binding site prediction. We previously used the first three terms for proteinprotein interface prediction (PINUP[29]). In an independent comparative study the PINUP method showed the highest prediction accuracy compared to other published interface prediction approaches [31]. The last three terms have already been used for antibody binding site prediction by others. We describe the details of those six terms in the following paragraphs.

\section{Residue epitope propensity}

The score of antibody binding site propensity, $E_{\text {propen- }}$ sity $(i)$, is defined as

$$
E_{\text {propensity }}(i)=\left(\ln \frac{P_{r}^{\text {interface }}}{P_{r}^{\text {surface }}}\right) \cdot \frac{S_{r}}{S_{r}^{\text {ave }}}
$$

Where $P_{\mathrm{r}}^{\text {interface }}$ and $P_{\mathrm{r}}^{\text {surface }}$ are the contribution of residue type $r$ to the antibody binding site and to the protein surface area, respectively, $S_{\mathrm{r}}$ and $S_{\mathrm{r}}^{\text {ave }}$ are the relative accessible surface area of residue $r$ at the sequence position $i$ and the average relative accessible surface area of surface residues of type $r$, respectively. The $\mathrm{C}_{\alpha}$ atom of Gly is considered as a side chain atom for convenience. Since antigen-antibody interfaces have different residue composition compared with other protein-protein interfaces, we used Protein Dataset 1 to derive residue antibody binding site propensity instead of using the former residue interface propensity score[29]. Here, $P_{\mathrm{r}}^{\text {interface }}$ and $P_{\mathrm{r}}^{\text {surface }}$ were obtained from statistical analysis of Protein Dataset 1. Some antigens in Dataset 1 have multiple epitopes. Those residues belonging to any of the epitopes were considered as antibody-binding interface residues. The values of $S_{\mathrm{r}}^{\text {ave }}$ for 20 amino acid residues were obtained from the statistical analyses on 41 antigens in Protein Dataset 1.

\section{Residue conservation score}

Residue conservation was measured by the self-substitution score from the sequence profile. Sequence profiles were obtained by three rounds of PSI-BLAST searches with the BLOSUM62[32] substitution matrix. The conservation score at the position $i$ is defined as

$$
E_{\text {conserv }}(i)=\left\{\begin{array}{c}
\left|M_{i r}-B_{r r}\right|, \text { if } M_{i r}-B_{r r}<0 \\
0, \text { if } M_{i r}-B_{r r}>0 .
\end{array}\right.
$$

where $M_{i r}$ is the self-substitution score in the position-specific substitution matrix generated from PSIBLAST for the residue type $r$ at sequence position $i$, and $B_{r r}$ is the diagonal element of BLOSUM62 for residue type $r$. Usually, protein-protein interface residues are more conserved than other surface residues due to functional constraints, and hence, the conserved surface residues in the unbound structure will be predicted as interface residues. The residues in the antibody-binding site, however, are less conserved than other surface residues due to the constraint of the host immune system. The unconserved residues are considered as the putative antibody binding site residues.

\section{Side-chain energy score}

The exact expression for side chain energy score can be found in Eq. (3) in PINUP [29]. It was calculated from the side-chain energies of all possible rotamers for a given residue type at a sequence position whereas other sequence positions have native residue types and observed atomic coordinates. The weights of the energy function were optimized so that the native residue was predicted energetically favorable at each position of the training proteins [33] The assumption is that the residues at the antibody binding site may have a higher energy score than other surface residues so that the free energy of the antigen-antibody system could go down significantly upon association.

\section{Contact number}

The residue contact number is the number of $\mathrm{C}_{\alpha}$ atoms in the antigen within a distance of $10 \AA$ of the $\mathrm{C}_{\alpha}$ atom of residue $i$ [20]. A residue with a small contact number was considered as an antibody binding site residue.

\section{Planarity score}

The planarity of each surface patch was calculated by evaluating the root mean squared (rms) deviation of all the $\mathrm{C}_{\alpha}$ atoms in the surface patch from the least squares plane through the atoms. The rms deviations were inverted such that a high planarity score for a patch was interpreted as a planar patch and antibody binding site[34]. 


\section{Secondary structure composition}

This score was defined as the fraction of patch residues forming turns or loops in all 20 patch residues. Following Chou \& Fasman's method[35], the $\alpha$-helix and $\beta$-sheet were defined as four or more consecutive residues having $\varphi, \psi$ angles within $40^{\circ}$ of $\left(-60^{\circ},-50^{\circ}\right)$ and three or more residues having $\varphi, \psi$ angles within $40^{\circ}$ of $\left(-120^{\circ}, 110^{\circ}\right)$ or $\left(-140^{\circ}, 135^{\circ}\right)$, respectively. The remaining regions were considered turns and loops.

\section{Prediction of discontinuous epitopes}

Prediction with one term

Given the structure of an antigen, all of its surface residues were sampled, and hence all its corresponding surface patches could be obtained. The score of a patch for one scoring function is given by the average value of its scores for all 20 residues. Based on a certain threshold, the central residue of the top percentile patch was predicted as an interface residue. In case of secondary structure composition and contact number score if a patch was not ranked above the threshold but scored the same as any top ranked patch, the patch was also added into the top-ranked patch set.

\section{Prediction with consensus scoring}

To take the advantage of the multiple features, we used a voting mechanism with the above described six scoring functions. A patch was considered as an interface patch if five of the all six terms scored it into the top-ranked patch set. We did not use the vote mechanism of all six votes from the six scoring function because one surface patch with a small contact number could not have a high planarity score at the same time. The number of predicted residues with each single term is the same but the threshold of how many top ranked patches shall be kept can be varied to yield predictions with different sensitivities.

\section{Patch score derived by unevenly averaged single-residue scores}

Patch analysis is widely used in protein binding site prediction. In general, it is assumed that each residue in the patch contributes equally to the patch score. Here, we exploited patch scoring with a weight decreasing with the distance from the center of the patch,

$$
E_{\text {patch }}(i)=\sum_{k=1}^{20} E_{\text {residue }}(k) \cdot e^{\frac{-d}{T}},
$$

where $E_{\text {residue }}(k)$ is the score of residue $k$ in the patch; $d$ is the distance between residue $k$ and the central residue of the patch; $T$ is the parameter to be optimized during training.

\section{Evaluation methods}

Sensitivity and precision were defined as the ratios of the number of correctly predicted interface residues to the number of real interface residues and to the number of all predicted interface residues, respectively. Specificity is defined as the fraction of correctly predicted surface residues in the total number of observed surface residues. As recommended by Ponomarenko \& Bourne[25], the area under the receiver operating characteristic curve (AUC) was used as the primary evaluation metric. A receiver operating characteristic (ROC) curve represents a dependency of sensitivity and (1-specificity). To obtain the ROC curve, we increased the number of predicted residues (or the predicted residue with the single term in consensus prediction) in steps of $1 \%$ of total surface residues. A java program downloaded from http://pages.cs.wisc.edu/ richm/programs/AUC/, was used to calculate the AUC.

\section{Results and Discussions Predictions with one term}

As a first test, we used each single term described in the Materials and Methods section to evaluate Protein Dataset 2 (training set) and Protein Dataset 3 (testing set). The residue antibody binding site propensity was independently derived from Protein Dataset 1 (Table 1). The correlation coefficient between the antibody binding site propensity and the protein-protein interface propensity values used in a previous study [29] is -0.15 . This indicates clearly that antigen-antibody complexes have unique interface properties and that propensity value specifically derived from antigen-antibody complexes should be used for antibody binding site prediction. Especially, cysteine has a exceptionally large negative value of ( $\left.\ln P_{\mathrm{r}}^{\text {interface }} / P_{r}^{\text {surface }}\right) / S_{\mathrm{r}}^{\text {ave }}$ because it is seldom found at antibody binding sites [20] and usually has a small solvent accessibility. On the other hand, cysteine is enriched relative to protein surfaces in general at other protein-protein interfaces[29] Excluding cystine, the correlation coefficient between the antibody binding site propensity and the protein-protein interface propensity rises to 0.3 .

Antibody binding site propensity alone results in a quite accurate prediction (AUC $=0.637$ ) for the training set (Protein Dataset 2). This is because the training set overlaps with Protein Dataset 1, which was used to derive the antibody binding site propensity values for the 20 amino acids. The prediction accuracy is considerably lower (0.577) for the testing set. It is possible that the propensity values were over trained. Other terms showed prediction accuracies only slightly better than random. Most of the contributing terms showed quite different performance for the training and testing sets (Table 2). For example, the score based on secondary structure composition showed no prediction ability for the training set, whereas it was the most effective term for the testing set. Antibody binding site propensity, conservation score, and contact 
Table I: The values of $\left(\ln P_{\mathrm{r}}^{\text {interface }} / P_{r}^{\text {surface }}\right) / S_{\mathrm{r}}^{\text {ave }}$ for twenty amino acid residues.

\begin{tabular}{llll}
\hline Amino Acid & $\left(\ln P_{\mathrm{r}}^{\text {interface }} / P_{r}^{\text {surface }}\right) / S_{\mathrm{r}}^{\text {ave }}$ & Amino Acid & $\left(\ln P_{\mathrm{r}}^{\text {interface }} / P_{r}^{\text {surface }}\right) / S_{\mathrm{r}}^{\text {ave }}$ \\
\hline Ala & -0.392 & Leu & -1.31 \\
Arg & 0.316 & Lys & 0.021 \\
Asn & 0.446 & Met & 1.06 \\
Asp & -0.307 & Phe & 0.979 \\
Cys & -7.36 & Pro & 0.017 \\
Gln & -0.006 & Trp & -0.07 \\
Glu & -0.492 & Val & -0.826 \\
Gly & 0.463 & Ser & -0.004 \\
His & 0.207 & Thr & -0.062 \\
Ile & 0.334 & Tyr & 0.979 \\
\hline
\end{tabular}

$P_{\mathrm{r}}^{\text {interface }}$ is the contribution of residue type $r$ to the area of antibody binding site; $P_{\mathrm{r}}^{\text {surface }}$ is the contribution of residue type $r$ to the protein

surface area; $S_{\mathrm{r}}^{\text {ave }}$ is the average relative accessible surface area of surface residues of type $r$.

number were the three most effective terms for the training set but were only medium predictors for the testing set.

\section{Prediction results for the training dataset with consensus scoring}

As shown in Table 2, all individual terms showed only slightly better prediction than a random prediction and the performance varies with the selected protein datasets. Combination of multiple features to increase the prediction accuracy is a challenge. Recently, Sweredoski \& Baldi concluded that non-linear methods such as SVMs, ANNs, and Gaussian Mixture Models did not achieve higher performance than a linear combination [36]. Here, we found that the precision of most terms does not increase for both training and testing sets as the number of predicted residues decreases except for conservation score (Fig 1). Selection of top scored residues by linear combination has no advantage over the consensus prediction of several terms.

Table 2: AUC values for training and testing datasets predicted by the single term

\begin{tabular}{lll}
\hline Evaluation terms & Training set & \\
\hline Binding site propensity & 0.637 & Testing set $^{b}$ \\
Conservation score & 0.593 & 0.577 \\
Side chain energy score & 0.555 & 0.564 \\
Contact number & 0.59 & 0.569 \\
Planarity score & 0.53 & 0.556 \\
Fraction of turns \& loops & 0.489 & 0.554 \\
\hline
\end{tabular}

a Antigen-antibody complexes from protein docking benchmark 2.0. b 17 recently released antigen-antibody complex structures in PDB. Unbound structures of both databsets were used for prediction and bound structures were used for identification of interface residues. The AUC values were calculated and averaged for all the proteins in two datasets, respectively.
In fact, when the contribution of the six terms was considered to be equal (as an example) and the sum of the six scores was used for re-ranking, we obtained an average AUC of 0.603 for the training set. When the residues, which were predicted as interface residues by 5 out of the 6 terms, were selected as the final prediction, the AUC value was 0.614 for the training set.

The precision of the conservation score always increases for both the training and testing sets as the number of predicted residues decreases. The residues scored above the cutoff value by only conservation were also considered as interface residues. Furthermore, when the predicted residues with the single term are less than $28 \%$ of total surface residues, none of interface residues are predicted by consensus scoring for at least one training protein and interface residues are only predicted by conservation score. We tried the cutoff value of $5 \%, 10 \%, 15 \%$, and $20 \%$, and the AUC values were $0.619,0.622,0.626$, and 0.618 respectively. The cutoff value (15\%) yielding the best AUC value was selected.

All the residues in the surface patch contributed equally to the patch score in the above predictions and only the central residues of the top scored patches were selected. In order to optimize the patch score with respect to the position of residues in the patch, the score of each patch residue was considered assuming an exponential decrease of the weight with the distance between patch residues and the central residue. The decay constant $T$ in equation (3) was allowed to take the values $4,8,12$, and 16 . The corresponding AUC values were 0.622, 0.633, 0.635, and 0.629 , respectively, for the training set. The $T$ value of 12 yielding the best AUC value was selected for applications on the test set. Table 3 lists the results for the training set. 

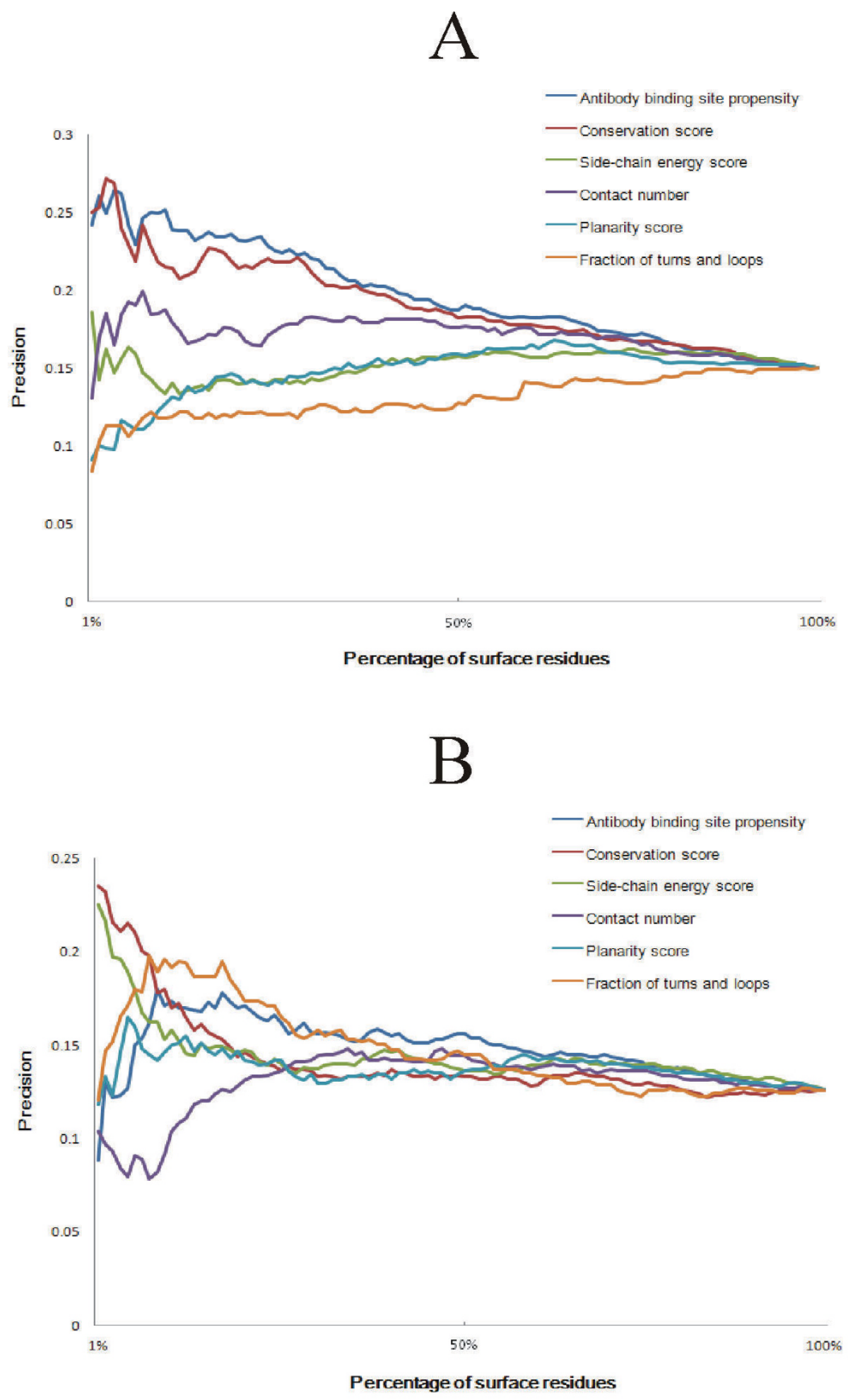

\section{Figure I}

Correlation between precision and the number of predicted residues (a) Training set; (b) Testing set. The prediction results of all the proteins in the datasets were calculated and averaged. The precisions of random prediction are $15 \%$ and $12.6 \%$ for the training and testing sets, respectively. 
Table 3: Prediction results for the training set with 6 combined terms

\begin{tabular}{|c|c|c|c|c|c|c|c|}
\hline Complex & Unbound & No. of Surface Residues & No. of Interface Residues & Sensitivity ${ }^{a}$ & Precisiona & Specificitya & $A \cup C$ \\
\hline $\mathrm{I} A H W \_\mathrm{AB}: \mathrm{C}$ & ITFH_A & 173 & 25 & 0.360 & 0.153 & 0.662 & 0.481 \\
\hline IBVK_DE:F & $3 \mathrm{LZT}_{-}$ & 98 & 17 & 0.765 & 0.361 & 0.716 & 0.835 \\
\hline IDQJ_AB:C & $3 \mathrm{LZT}_{-}$ & 98 & 20 & 0.300 & 0.182 & 0.654 & 0.534 \\
\hline IE6]_HL:P & IA43_ & 63 & 13 & 0.462 & 0.353 & 0.780 & 0.585 \\
\hline IJPS_HL:T & ITFH_B & 155 & 25 & 0.360 & 0.170 & 0.662 & 0.517 \\
\hline I MLC_AB:E & $3 \mathrm{LZT}_{-}^{-}$ & 98 & 16 & 0.562 & 0.250 & 0.671 & 0.636 \\
\hline IVFB_AB:C & $8 \mathrm{LYZ}$ & 107 & 18 & 0.833 & 0.385 & 0.730 & 0.833 \\
\hline IWEJ_HL:F & IHRC_ & 95 & 13 & 0.462 & 0.188 & 0.683 & 0.649 \\
\hline 2VIS_AB:C & 2VIU_A & 247 & 20 & 0.900 & 0.281 & 0.797 & 0.901 \\
\hline IBJI_HLJK:VWb & 2VPF_GH & 160 & 35 & 0.600 & 0.412 & 0.760 & 0.705 \\
\hline IFSK_BC:A & $\mid B V I_{-}$ & 145 & 19 & 0.526 & 0.233 & 0.738 & 0.587 \\
\hline II & IALY_ABC & 320 & 65 & 0.508 & 0.292 & 0.686 & 0.687 \\
\hline IIQD_AB:C & ID7P_M & 127 & 17 & 0.765 & 0.361 & 0.791 & 0.848 \\
\hline IK4C_AB:C & IJVM_A & 88 & 16 & 0.500 & 0.258 & 0.681 & 0.647 \\
\hline IKXQ_H:A & IPPI_ & 341 & 30 & 0.600 & 0.148 & 0.666 & 0.637 \\
\hline INCA_HL:N & 7NN9_ & 263 & 27 & 0.556 & 0.163 & 0.674 & 0.684 \\
\hline INSN_HL:S & $\mathrm{IKDC}_{-}$ & 106 & 23 & 0.174 & 0.114 & 0.627 & 0.454 \\
\hline IQFW_HL:AB & IHRP_AB & 170 & 17 & 0.235 & 0.071 & 0.660 & 0.484 \\
\hline IQFW_IM:AB & IHRP_AB & 170 & 17 & 0.706 & 0.214 & 0.712 & 0.738 \\
\hline 2JEL_HL:P & $\mathrm{IPOH}_{-}$ & 68 & 18 & 0.167 & 0.158 & 0.680 & 0.498 \\
\hline IBGX_HL:T & ICMW_A & 646 & 66 & 0.394 & 0.124 & 0.683 & 0.521 \\
\hline 2HMI_CD:AB & IS6P_AB & 810 & 14 & 0.429 & 0.024 & 0.697 & 0.518 \\
\hline Mean & & 207 & 24.1 & 50.7 & 0.222 & 0.7 & 0.635 \\
\hline
\end{tabular}

a Sensitivity, precision, and specificity were recorded when $55 \%$ of surface residues were predicted as interface residues by the single term. We chose the parameter (55\%) so that the sensitivity was about $50 \%$ in the consensus prediction. bMultiple binding sites.

\section{Independent test}

The average AUC value was 0.616 for the testing set when the residues predicted as interface residues by 5 of the 6 terms were selected in the consensus prediction. The AUC value increased to 0.621 when the residues top scored by conservation were included in the prediction (cutoff value $=15 \%$ ) and further increased to 0.632 (Table 4) if non- uniform averaged patch scores were used $(T=12)$. The prediction accuracy was very close to that for the training set (0.635). Fig. 2 shows two successful predictions. The real antibody binding sites overlapped with the largest cluster of red colored residues which correspond to the predicted antigenic epitope residues.

Table 4: Prediction results for the testing set

\begin{tabular}{|c|c|c|c|c|c|c|c|}
\hline Complex & Unbound & No. of Surface Residues & No. of Interface Residues & Sensitivitya & Precisiona $^{a}$ & Specificity ${ }^{a}$ & $A \cup C$ \\
\hline 2ARJ_HL:Q & INEZ_G & 99 & 18 & 0.278 & 0.227 & 0.790 & 0.604 \\
\hline 2BDN_HL:A & IDOK_A & 63 & 13 & 0.154 & 0.095 & 0.620 & 0.281 \\
\hline 2FD6_HL:U & IYWH_A & 225 & 14 & 0.500 & 0.089 & 0.659 & 0.617 \\
\hline 2GHW_B:A & $2 \mathrm{GHV} \_\mathrm{E}$ & 148 & 27 & 0.519 & 0.311 & 0.744 & 0.727 \\
\hline 2H9G_AB:R & ID4V_A & 108 & 18 & 0.556 & 0.286 & 0.722 & 0.724 \\
\hline 2J6E_IMHL:ABb & 2DTQ_AB & 336 & 41 & 0.512 & 0.202 & 0.719 & 0.614 \\
\hline 2NR6_CD:A & IYG9_A & 233 & 19 & 0.947 & 0.234 & 0.724 & 0.870 \\
\hline 2NYY_CD:A & 2VUA_A & 321 & 24 & 0.750 & 0.164 & 0.690 & 0.810 \\
\hline 2P45_B: $\mathrm{B}: \mathrm{A}$ & IKF2_A & 104 & 13 & 0.154 & 0.057 & 0.637 & 0.553 \\
\hline 2Q8B_HL:A & IZ40_A & 228 & 25 & 0.440 & 0.147 & 0.685 & 0.645 \\
\hline 2QQN_HL:A & IKEX_A & 118 & 11 & 0.636 & 0.200 & 0.738 & 0.737 \\
\hline 2R29_HL:A & IOK8_A & 317 & 20 & 0.300 & 0.054 & 0.646 & 0.567 \\
\hline 2R56_HL:A & IGX9_A & $13 \mid$ & 22 & 0.091 & 0.053 & 0.670 & 0.409 \\
\hline 2UZI_HL:R & 2EVW_X & 132 & 21 & 0.286 & 0.171 & 0.739 & 0.505 \\
\hline 3BN9_CD:B & IEAX_A & $|8|$ & 32 & 0.531 & 0.279 & 0.705 & 0.581 \\
\hline 3BQU_CD:AB & 2F5A_HL & 336 & 12 & 1.000 & 0.098 & 0.660 & 0.914 \\
\hline 3D85_AB:C & 3D87_A & $14 \mid$ & 19 & 0.474 & 0.180 & 0.664 & 0.591 \\
\hline Mean & & 189 & 20.5 & $47.8 \%$ & 16.7 & $69.5 \%$ & 0.632 \\
\hline
\end{tabular}

a Sensitivity, precision, and specificity were recorded when $55 \%$ of surface residues were predicted as interface residues by the single term. bMultiple binding sites. 


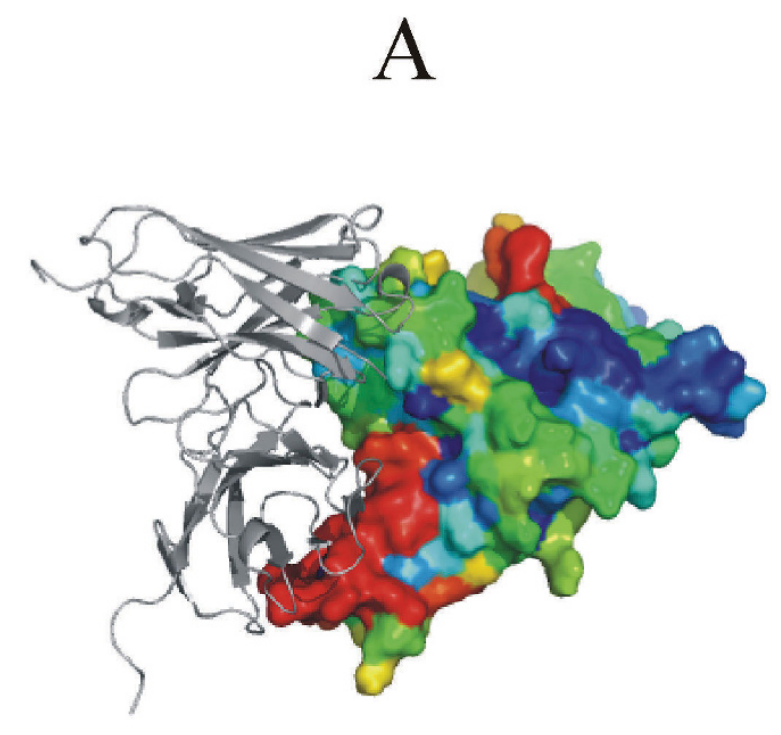

B

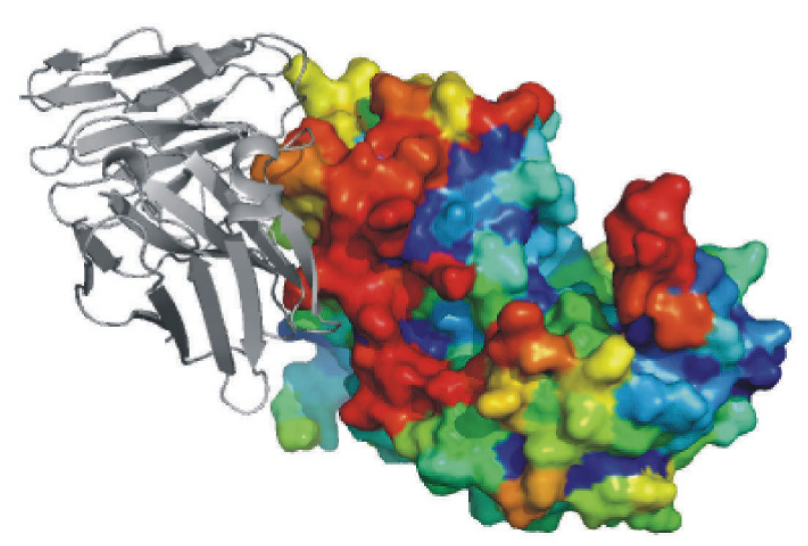

Figure 2

Two successful examples of antibody binding site prediction (a) SARS spike protein receptor binding domain (2ghv); (b) Cockroach allergen Bla g 2 (lyg9). The antibodies were colored in grey. The surface residues of antigens were colored according to predicted possibility to be an epitope residue (from red to blue in decreasing order) and the core residues were colored in blue.

\section{Comparison with other epitope prediction methods}

In this study, we investigated residue antibody binding site propensity based on atomic solvent accessibility for 20 amino acids. The AUC values for training and testing sets were 0.637 and 0.577 , respectively, when the single term of propensity score were used for prediction. Currently, two other algorithms using multiple features for antibody binding site prediction are available, Disco-
Tope[20] and PEPITO[36]. These methods used similar antibody binding site propensity scores at residue level. We also tried using the propensity score of DiscoTope [20] for comparative predictions. The AUC values were 0.587 and 0.551 for our training and testing sets, respectively. Propensity score based on atomic solvent accessibility has a slightly better performance than the propensity score of residue level for both datasets.

We compared our consensus algorithm with the recently updated version of DiscoTope[20] and PEPITO[36], DiscoTope 1.2 http://www.cbs.dtu.dk/services/DiscoTope and BEpro http://scratch.proteomics.ics.uci.edu. The computation was conducted on the websites for each method between December 2008 and January 2009. As shown in Table 5, all of the algorithms have similar prediction for the training set while our algorithm showed a better prediction (AUC $=0.632$ ) for the unbound structures of testing set than DiscoTope (0.589) and BEpro (0.598). It should be noted that all the PDB files in the testing set were released very recently so these structures were not part of the training set for the two published methods and could serve as independent testing cases for other algorithms as well. In the original paper an AUC value of 0.71 was reported for DiscoTope averaged over five evaluation sets used for cross validation [20]. However, a considerable smaller AUC value for DiscoTope of 0.566 for 30 targets out of 59 representative antigen-antibody complexes, which were compiled by Ponomarenko \& Bourne and not used for training DiscoTope was reported in a recent study [25]. The prediction accuracy of recently released DiscoTope 1.2 was slightly improved compared with the original DiscoTope in the two independent tests.

Unlike Discotop1.2 and BEpro, our algorithm has lower prediction accuracy for the bound structures than the unbound structures due to the inclusion of the side chain energy score. The interface residues of bound antigen are buried in the complex and usually have a lower temperature factor than other surface residues. In the bound forms these side chains have systematically lower energies than in the unbound form which in our algorithm contributes unfavorably to the score [27]. Predictions with the side chain energy score as single term yielded AUC values of training and testing sets of 0.555 and 0.569 , respectively, for unbound structures and 0.532 and 0.521 for bound structures, respectively.

\section{Conclusion}

An important conclusion of the present study is that antibody binding site prediction is more difficult than prediction of other protein binding regions. A combination of multiple surface features which allows relatively accurate prediction of protein binding sites in general shows 
Table 5: Comparison with other algorithms

\begin{tabular}{lcccc}
\hline Methods & \multicolumn{2}{c}{ Training set } & \multicolumn{2}{c}{ Testing set } \\
& Bound & Unbound & Bound & Unbound \\
\hline DiscoTopel.2 & 0.63 & 0.628 & 0.6 & 0.589 \\
BEpro & 0.645 & 0.639 & 0.617 & 0.598 \\
Our algorithm & 0.628 & 0.635 & 0.603 & 0.632 \\
\hline
\end{tabular}

poorer performance in case of antibody binding site prediction. An important issue is also that a given protein usually contains not only one but several putative antibody binding sites. Usually an antibody-antigen complex structure indicates only one of these possible antigenic epitopes. In addition, care must be taken in evaluating prediction methods when a relatively small number of antibody-antigen complexes were used as the testing set. The prediction algorithm may work reasonably well on one testing set but could show poorer prediction accuracy on new targets due to different interface properties. More training proteins are required for developing new prediction algorithms in the future. Nevertheless, the study demonstrated that consensus scoring of widely used features for binding site prediction showed a better performance than any single term for the independent test set. The prediction accuracy was improved further by utilizing residue epitope propensity based on atomic solvent accessibility. However, a detailed comparison with other published methods indicated that overall the performance of our combined approach is similar to existing methods.

\section{Availability}

The EPCES program is available upon request. A webbased EPCES application is available at: http:// www.t38.physik.tu-muenchen.de/programs.htm.

\section{Authors' contributions}

SL designed the study, implemented the algorithm and drafted the manuscript. DZ and CZ helped prepare the data, write the software and draft the manuscript. $M Z$ supervised the study. All authors read and approved the final manuscript.

\section{Acknowledgements}

We thank Dr. S. Fiorucci for helpful discussions. This project was supported by funding under the Sixth Research Framework Programme of the European Union (FP6 STREP "BacAbs", ref. LSHB-CT-2006-037325). Calculations were performed using computational resources of the CLAMV (Computational Laboratories for Analysis, Modeling and Visualization) at Jacobs University Bremen, Germany.

\section{References}

I. Van Regenmortel MHV: Mapping Epitope Structure and Activity: From One-Dimensional Prediction to Four-Dimensional Description of Antigenic Specificity. Methods 1996, 9:465-472.
2. Parker JM, Guo D, Hodges RS: New hydrophilicity scale derived from high-performance liquid chromatography peptide retention data: correlation of predicted surface residues with antigenicity and X-ray-derived accessible sites. Biochemistry 1986, 25:5425-5432.

3. Hopp TP, Woods KR: Prediction of protein antigenic determinants from amino acid sequences. Proc Natl Acad Sci USA I 98I, 78:3824-3828.

4. Emini EA, Hughes JV, Perlow DS, Boger J: Induction of hepatitis A virus-neutralizing antibody by a virus-specific synthetic peptide. J Virol 1985, 55:836-839.

5. Pellequer JL, Westhof E, Van Regenmortel MH: Correlation between the location of antigenic sites and the prediction of turns in proteins. Immunol Lett 1993, 36:83-99.

6. Karplus PA, Schulz GE: Prediction of Chain Flexibility in Proteins a -Tool for the Selection of Peptide Antigens. Naturwissenschaften 1985, 72:212-213.

7. Kolaskar AS, Tongaonkar PC: A semi-empirical method for prediction of antigenic determinants on protein antigens. FEBS Lett 1990, 276: 172-174.

8. Saha S, Bhasin M, Raghava GP: Bcipep: a database of B-cell epitopes. BMC Genomics 2005, 6:79.

9. Schonbach C, Koh JL, Sheng X, Wong L, Brusic V: FIMM, a database of functional molecular immunology. Nucleic Acids Res 2000, 28:222-224.

10. Larsen JE, Lund O, Nielsen M: Improved method for predicting linear B-cell epitopes. Immunome Res 2006, 2:2.

II. Saha S, Raghava GP: Prediction of continuous B-cell epitopes in an antigen using recurrent neural network. Proteins 2006, 65:40-48.

12. Chen J, Liu H, Yang J, Chou KC: Prediction of linear B-cell epitopes using amino acid pair antigenicity scale. Amino Acids 2007, 33:423-428.

13. El-Manzalawy Y, Dobbs D, Honavar V: Predicting linear B-cell epitopes using string kernels. J Mol Recognit 2008, 2I:243-255.

14. Blythe MJ, Flower DR: Benchmarking B cell epitope prediction: underperformance of existing methods. Protein Sci 2005, 14:246-248

15. Greenbaum JA, Andersen PH, Blythe M, Bui HH, Cachau RE, Crowe J, Davies M, Kolaskar AS, Lund O, Morrison S, et al:: Towards a consensus on datasets and evaluation metrics for developing $B$ cell epitope prediction tools. J Mol Recognit 2007, 20:75-82

16. Peters B, Sidney J, Bourne P, Bui HH, Buus S, Doh G, Fleri W, Kronenberg $M$, Kubo $R$, Lund $O$, et al.: The immune epitope database and analysis resource: from vision to blueprint. PLoS Biol 2005, 3:e91.

17. Allcorn LC, Martin AC: SACS--self-maintaining database of antibody crystal structure information. Bioinformatics 2002 18: $175-181$

18. Huang J, Honda W: CED: a conformational epitope database. BMC Immunol 2006, 7:7.

19. Kulkarni-Kale U, Bhosle S, Kolaskar AS: CEP: a conformational epitope prediction server. Nucleic Acids Res 2005, 33:WI68-17I.

20. Andersen PH, Nielsen M, Lund $O$ : Prediction of residues in discontinuous B-cell epitopes using protein 3D structures. Protein Science 2006, 15:2558-2567.

21. Rapberger R, Lukas A, Mayer B: Identification of discontinuous antigenic determinants on proteins based on shape complementarities. J Mol Recognit 2007, 20: I I3-12I.

22. Caoili SE: A structural-energetic basis for B-cell epitope prediction. Protein Pept Lett 2006, I3:743-75 I.

23. Rubinstein ND, Mayrose I, Pupko T: A machine-learning approach for predicting B-cell epitopes. Mol Immunol 2009, 46:840-847.

24. Ponomarenko J, Bui HH, Li W, Fusseder N, Bourne PE, Sette $A$, Peters B: ElliPro: a new structure-based tool for the prediction of antibody epitopes. BMC Bioinformatics 2008, 9:5।4.

25. Ponomarenko JV, Bourne PE: Antibody-protein interactions: benchmark datasets and prediction tools evaluation. $B M C$ Struct Biol 2007, 7:64.

26. Mintseris J, Wiehe K, Pierce B, Anderson R, Chen R, Janin J, Weng Z: Protein-Protein Docking Benchmark 2.0: an update. Proteins 2005, 60:214-216.

27. Liang S, Zhang J, Zhang S, Guo H: Prediction of the interaction site on the surface of an isolated protein structure by analysis of side chain energy scores. Proteins 2004, 57:548-557. 
28. Jones S, Thornton JM: Analysis of protein-protein interaction sites using surface patches. J Mol Biol 1997, 272:121-132.

29. Liang S, Zhang C, Liu S, Zhou Y: Protein binding site prediction using an empirical scoring function. Nucleic Acids Res 2006, 34:3698-3707.

30. Jones S, Thornton JM: Prediction of protein-protein interaction sites using patch analysis. Journal of Molecular Biology 1997, 272:133-143.

31. Zhou HX, Qin S: Interaction-site prediction for protein complexes: a critical assessment. Bioinformatics 2007, 23:2203-2209.

32. Henikoff $S$, Henikoff JG: Amino acid substitution matrices from protein blocks. Proc Natl Acad Sci USA 1992, 89:109/5-10919.

33. Liang S, Grishin NV: Effective scoring function for protein sequence design. Proteins 2004, 54:27|-28I.

34. Jones S, Thornton JM: Analysis of protein-protein interaction sites using surface patches. Journal of Molecular Biology 1997, 272: $121-132$.

35. Chou PY, Fasman GD: Empirical predictions of protein conformation. Annu Rev Biochem 1978, 47:25 I-276.

36. Sweredoski MJ, Baldi P: PEPITO: improved discontinuous B-cell epitope prediction using multiple distance thresholds and half sphere exposure. Bioinformatics 2008, 24:|459-1460.

Publish with Bio Med Central and every scientist can read your work free of charge

"BioMed Central will be the most significant development for disseminating the results of biomedical research in our lifetime. "

Sir Paul Nurse, Cancer Research UK

Your research papers will be:

- available free of charge to the entire biomedical community

- peer reviewed and published immediately upon acceptance

- cited in PubMed and archived on PubMed Central

- yours - you keep the copyright

Submit your manuscript here:

http://www.biomedcentral.com/info/publishing_adv.asp
BiolMedcentral 